\title{
ON THE NOMENCLATURE OF GOTTSCHEA NEES EX MONT. 1843 (SCHISTOCHILACEAE, JUNGERMANNIALES)
}

\author{
Riclef Grolle' and Gea Zijlstra
}

\section{Summary}

Gottschea Nees ex Mont. 1843 has been published without generic description. It is valid there by indirect reference to Jungermannia sect. Nemorosae [subsect.] Aligerae Reinw. et al. 1824 only. The type of both is J. aligera Nees et Blume (Art. 22.4). Schistochilaster H. Miller 1970 is a nomenclatural synonym and Paraschistochila Schust. 1963 and Tegulifolium Hässel 1973 are taxonomic ones. 18 new combinations are proposed under Gottschea.

Schistochila Dum. 1835 s. amplo has recently been split into several genera (Schuster, 1963; Miller, 1970; Hässel de Menendez, 1973; Schuster and Engel, 1982). This raised the question, whether the synonyms of Schistochila s. amplo have to be kept as those of Schistochila s. str. as well.

Following Miller's 1970 nomenclatural treatment of Gottschea Nees ex Mont. 1843, Schuster (1971, p. 625 [footnote]) concluded that "there is no Schistochila-Gottschea problem concerning nomenclature."

A recent checking of the protologue of Gottschea in Montagne (1843) revealed however that Miller's 1970 typification of Gottschea Nees ex Mont. 1843 is untenable. The reasons are the following:

Montagne (1843) described three new species of Gottschea, and mentioned three further species. The study of the fructification of $G$. neesii made him conclude that the hepatics of the "section Aligerae" should be considered as a genus of its own. He wrote this to Nees, who answered him that he had come to the same conclusions, and was publishing this now as the n.g. Gottschea in the first fascicule of the 'Synopsis Hepaticarum' (which appeared in 1844). Montagne did not provide a generic diagnosis, but Gottschea is validly published by him, by his indirect reference to the "section Aligerae." Hence J. aligera, the type of the Aligerae, is the type of Gottschea (Art. 22.4).

With Jungermannia aligera Nees et Blume as the type of Gottschea Nees ex Mont. 1843, this generic name has to replace Paraschistochila Schust. 1963 following the taxonomic concept advocated by Schuster (1971) and Schuster and Engel (1977). Unfortunately, 18 new combinations are required. The name commemorates, however, C. M. Gottsche (1808-1892), an eminent expert of hepatics in the past century and physician to the poor in Hamburg.

Gottschea Nees ex Mont., Ann. Sci. Nat., Bot., ser. 2, 19: 245. Apr 1843. Type (Art. 7.9): G. aligera (Nees et Blume) Nees (Jungermannia a. Nees et Blume). Based on Jungermannia sect. Nemorosae [subsect.] Aligerae Reinw. et al., Nova Acta Acad. Caes. Leop. 12: 189, 234. 1824. Nees, Enumeratio Pl. Crypt. Jav. 66; 15 Sep-31 Dec 1830. Type (Art. 22.4): Jungermannia aligera Nees et Blume. - Schistochilaster H. Miller, Phytologia 20: 317. 4 Sep 1970; nom. illeg. (Art. 63.1). Type: S. aliger (Nees et Blume) H. Miller ("aligerum") (Jungermannia a. Nees et Blume).

=Paraschistochila Schust., Journ. Hattori Bot. Lab. 26: 259. "Jul" 29 Aug 1963. Type: P. pinnatifolia (Hook.) Schust. (Jungermannia p. Hook.).

1 Sektion Biologie, Friedrich-Schiller-Universität, Goetheallee 26, DDR-69 Jena.

2 Instituut voor Systematische Plantkunde, Heidelberglaan 2, 3584 CS Utrecht, The Netherlands. 
=Tegulifolium Hăssel. Boletim Soc. Argent. Bot. 15: 252. Dec 1973. Type: T. spegazzinianum (Mass.) Hässel (Gottschea s. Mass.)

G. subg. Nothoschistochila (Schust.) comb. nov.

= Paraschistochila subg. Nothoschistochila Schust., Bull. Nat. Sci. Mus. Tokyo 14: 642. 1971. Type: G. spegazziniana Mass.

G. spegazziniana Mass., Nuovo Giom. Bot. Ital. 17: 206. 1885. Schistochilaster S. (Mass.) H. Miller, Phytologia 20: 319. 1970. Paraschistochila s. (Mass.) Schust., Bull. Nat. Sci. Mus. Tokyo 14:643. 1971. Tegulifolium s. (Mass.) Hässel. Boletim Soc. Argent. Bot. 15: 252. 1973.

G. subg. Paraschistochila (Schust.) stat. et comb. nov.

= Paraschistochila Schust.. Journ. Hattori Bot. Lab. 26: 259. 1963. Schistochila sect. Paraschistochila (Schust.) Hamlin. Records Dominion Mus. (Wellington) 7: 333. 1972. Type: G. pinnatifolia (Hook.) Nees (Jungermannia p. Hook.).

- (by lectotypification) Gottschea sect. Complicatae Gott. et al., Synop. Hepat.: 21. 1844. Schistochila sect. Complicatae (Gott. et al.) Schiffn. in Engler et Prantl, Natürl. Pflanzenfam. 1(3): 111. 1893. Lectotype (as a residue in Schiffner, 1893: III): G. pinnatifolia (Hook.) Nees (Jungermannia p. Hook.).

G. pinnatifolia (Hook.) Nees in Gott. et al.. Synop. Hepat. 22. 1844. Jungermannia p. Hook.. Musci Exot. 2: 114. 1819 . Schistochila p. (Hook.) Trev., Memorie Real. Istit. Lombard. Sci. Mat. Nat. ser. 3, 4: 392. 1877. Paraschistochila p. (Hook.) Schust., Journ. Hatton Bot. Lab. 26: 259. 1963. - Gottschea pinnatifida Goebel. Flora (Jena) 96: 101. 102. Fig. 70. 1906 (Schuster. 1963, p. 259).

- Gottschea plumulosa Col.. Trans. Proc. New Zeal. Inst. 20: 249. 1888 (Stephani, 1892, p. 274: Hodgson, 1941, p. 190).

G. subg. Gottschen

= Paraschistochila subg. Acroschistochila Schust., Bull. Nat. Sci. Mus. Tokyo 14: 647. 1971. Type: G. conchophylla (Hodgs. et Allison) comb. nov. (Schistochila c. Hodgs. et Allison).

G. sect. Acroschistochila (Schust.) stat. et comb. nov.

- Paraschistochila subg. .tcroschistochila Schust., Bull. Nat. Sci. Mus. Tokyo 14: 647. 1971. Type: G. conchophylla (Hodgs. et Allison) comb. nov. (Schistochila c. Hodgs. et Allison).

G. conchophylla (Hodgs. et Allison) comb. nov. Schistochila conchophylla Hodgs. et Allison in Hodgson. Trans. Roy. Soc. New Zealand 71: 191. 1941 ("Schistophylla"). Schistochilaster c. (Hodgs. et Allison) H. Miller. Phytologia 20: 318. 1970. Paraschistochila c. (Hodgs. et Allison) Schust.. Bull. Nat. Sci. Mus. Tokyo 14: 647. 1971.

G. sect. Gortschea

- Jungermannia sect. Nemorosae [subsect.] .Aligerae Reinw. et al.. Nova Acta Acad. Caes. Leop. 12: 189. 234. 1824. Type (Art. 22.4): J. aligera Nees et Blume.

- Gottschea sect. Dorsilobae Gott. et al., Synop. Hepat.: 14. 1844: nom. illeg.: Art. 63.1. Type (Art. 7.11): G. aligera (Nees et Blume) Nees (Jungermannia a. Nees et Blume).

-Schistochila sect. Dorsilobae Schiffn. in Engler et Prantl. Natürl. Pflanzenfam. 1(3): 111. 1893. Type (An. 7.9): S. aligera (Nees et Blume) Jack et Steph. (Jungermannia a. Nees et Blume).

-Schistochila sect. Philippinenses Grolle. Journ. Hattori Bot. Lab. 29: 250. 1960. Paraschistochila sect. Philippinenses (Groile) Schust., Bull. Nat. Sci. Mus. Tokyo 14: 647. 1971 ("Philippensis"). Type: S. philippinensis (Mont.) Jack et Steph. (Gottschea p. Mont.).

= Paraschistochila sect. Tuloides Schust. Bull. Nat. Sci. Mus. Tokyo 14: 647. 1971. Type: P. tuloides (Hook. f. et Tayl.) Schust. (Jungermannia t. Hook. f. et Tayl.).

G. aligera (Nees et Blume) Nees in Gott. et al.. Synop. Hepat.: 17. 1844. Jungermannia a. Nees et Blume in Nees. Nova Acta Acad. Caes. Leop. 11: 135. 1823. Schistochila a. (Nees et Blume) Jack et Steph., Hedwigia 31: 12. 1892. Schistochilaster a. (Nees et Blume) H. Miller, Phytologia 20: 318. 1970. Paraschistochila a. (Nees et Blume) Schust., Bull. Nat. Sci. Mus. Tokyo 14: 650. 1971. -Schistochila curtisii Steph., Spec. Hep. 4: 77. 1909. Schistochilaster c. (Steph.) H. Miller. Phytologia 20: 318. 1970. (Kitagawa, 1973. p. 270). 
$=$ Schistochila cuspidata Steph., Spec. Hep. 4: 79. 1909. Paraschistochila c. (Steph.) Schust., Bull. Nat. Sci. Mus. Tokyo 14: 651. 1971. (Buch, 1939, p. 5).

=Schistochila maxima Steph., Spec. Hep. 6: 493. 1924. Schistochilaster m. (Steph.) H. Miller, Phytologia 20: 319. 1970. (Kitagawa, 1973, p. 270).

(Further synonyms are listed by Buch, 1939 and Grolle, 1966).

G. aligeriformis De Not., Memorie Real. Accad. Sci. Torino ser. 2, 28: 8.1874 ("aligeraeformis"). Schistochila a. (De Not.) Schiffn., Conspectus Hep. Arch. Ind.: 213. 1898. Schistochilaster a. (De Not.) H. Miller, Phytologia 20: 317. 1970.

G. brassii (Grolle) comb. nov.

Schistochila brassii Grolle, Journ. Hattori Bot. Lab. 31: 5. 1968.

G. brotheri (Steph.) comb. nov.

Schistochila brotheri Steph., Spec. Hep. 4: 85. 1909. Schistochilaster b. (Steph.) H. Miller, Phytologia 20: 318. 1970.

G. cheesemanii (Steph.) comb. nov.

Schistochila cheesemanii Steph., Spec. Hep. 4: 96.1909 ("cheesemani"). Schistochilaster c. (Steph.) H. Miller, Phytologia 20: 318. 1970. Paraschistochila c. (Steph.) Schust., Bull. Nat. Sci. Mus. Tokyo 14: 651. 1971.

G. crinita (Grolle) comb. nov.

Schistochila crinita Grolle, Journ. Hattori Bot. Lab. 31: 7. 1968.

G. cuspidiloba (Steph.) comb. nov.

Schistochila cuspidiloba Steph., Spec. Hep. 4: 82. 1909. Schistochilaster c. (Steph.) H. Miller, Phytologia 20: 318. 1970. Paraschistochila c. (Steph.) Schust., Bull. Nat. Sci. Mus. Tokyo 14: 651. 1971.

G. engleriana (Steph.) comb. nov.

Schistochila engleriana Steph., Spec. Hep. 4: 69. 1909. Schistochilaster e. (Steph.) H. Miller, Phytologia 20: 318. 1970. Paraschistochila e. (Steph.) Schust., Bull. Nat. Sci. Mus. Tokyo 14: 650. 1971.

G. fleischeri (Steph.) comb. nov.

Schistochila fleischeri Steph., Spec. Hep. 4: 81. 1909. Schistochilaster f. (Steph.) H. Miller, Phytologia 20: 318. 1970. Paraschistochila f. (Steph.) Schust., Bull. Nat. Sci. Mus. Tokyo 14: 651. 1971.

G. fragilis (Steph.) comb. nov.

Schistochila fragilis Steph., Spec. Hep. 4: 84. 1909. Schistochilaster f. (Steph.) H. Miller, Phytologia 20: 318. 1970.

G. gaudichaudii Gott., Ann. Sci. Nat., Bot. ser. 4, 8: 319. 1857. Schistochila g. (Gott.) Schiffn., Conspectus Hep. Arch. Indici: 216. 1898. Paraschistochila 8. (Gott.) Schust., Bull. Nat. Sci. Mus. Tokyo 14: 651. 1971.

G. graeffeana (Jack et Steph.) comb. nov.

Schistochila graeffeana Jack et Steph., Bot. Centralbl. 60: 97.1894 ("Schistocheila"). Schistochilaster g. (Jack et Steph.) H. Miller, Phytologia 20: 318. 1970. Paraschistochila g. (Jack et Steph.) Schust., Bull. Nat. Sci. Mus. Tokyo 14: 651. 1971.

G. integerrima (Steph.) comb. nov.

Schistochila integerrima Steph., Spec. Hep: 6: 492. 1924. Schistochilaster i. (Steph.) H. Miller, Phytologia 20: 318. 1970.

G. lacerata (Steph.) comb. nov.

Schistochila lacerata Steph., Spec. Hep. 6: 492. 1924. Schistochilasterl. (Steph.) H. Miller, Phytologia 20: 319. Paraschistochila I. (Steph.) Schust., Bull. Nat. Sci. Mus. Tokyo 14: 650. 1971.

G. neesii Mont., Ann. Sci. Nat., Bot. ser. 2, 19: 244. 1843. Schistochila n. (Mont.) Lindb., Journ. Linn. Soc., Bot. 13: 194. 1872. Schistochilaster n. (Mont.) H. Miller, Phytologia 20: 319. 1970. Paraschistochila n. (Mont.) Schust., Bull. Nat. Sci. Mus. Tokyo 14: 650. 1971.

= Gottschea schwaegricheniana Nees et Mont. in Mont., Ann. Sci. Nat., Bot. Ser. 2, 19: 244. 1843; nom. inval.: Art. 34(3) (pro syn.); Bonner, Index Hepat. 6: 500. 1966; nom. inval.: Art. 32.1(c).

-Schistochila borbonica Steph., Bot. Gazette 15: 290. 1890 ("Schistocheila"). Gottschea b. (Steph.) Bonner, Index Hepat. 6: 488. 1966. (Jones, 1976, p. 38).

G. nuda (Horik.) comb. nov.

Schistochila nuda Horik., Journ. Sci. Hiroshima Univ., ser. B, 2(2): 38. 1934. Paraschistochila n. (Horik.) Inoue, Illust. Jap. Hep. 2: 179. 1976.

G. philippinensis Mont., Ann. Sci. Nat., Bot. ser. 2, 19: 244. 1843. Schistochila p. (Mont.) Jack et 
Steph.. Bot. Centralbl. 60: 98.1894 ("Schistocheila"). Schistochilaster p. (Mont.) H. Miller. Phytologia 20: 319. 1970. Paraschistochila p. (Mont.) Schust., Bull. Nat. Sci. Mus. Tokyo 14: 647. 1971 ("philippensis").

-Schistochila linearifolia Jack et Steph., Bot. Centralbl. 60: 98. 1894 ("Schistocheila"). Schistochi. laster I. (Jack et Steph.) H. Miller. Phytologia 20: 319. 1970. Paraschistochila I. (Jack et Steph.) Schust.. Bull. Nat. Sci. Mus. Tokyo 14: 651. 1971. (syn. nov.; isotype in BM!. W!).

-Schistochila commutata Steph.. Spec. Hep. 4: 74. 1909. Paraschistochila c. (Steph.) Schust.. Bull. Nat. Sci. Mus. Tokyo 14: 650. 1971. (Buch. 1939. p. 7).

-Schistochila sumatrana Steph.. Spec. Hep. 4: 74. 1909. Paraschistochila s. (Steph.) Schust.. Bull. Nat. Sci. Mus. Tokyo 14: 650. 1971. (Buch. 1939. p. 7).

(Further synonyms are listed by Buch, 1939 and Grolle. 1966).

G. sphagnoides (Schwägr.) Lindb., Öfvers. Finska Vet.-Soc. Förhandl. 12: 82. 1870. Jungermannia s. Schwägr.. Hist. Musc. Hep. Prodromus: 23. 1814. Schistochila s. (Schwägr.) Steph., Spec. Hep. 4: 70. 1909.

= Jungermannia thouarsii Hook., Musci Exot. 1: tab. 48. 1818. Gottschea t. (Hook.) Nees in Gott. et al., Synop. Hepat.: 15. 1844. Schistochila t. (Hook.) Trev.. Memorie Real. Istit. Lombard. Sci. Mat. Nat. ser. 3. 4: 392. 1877. (Stephani. 1909. p. 70).

G. spinose (Steph.) comb. nov.

Schistochila spinosa Steph.. Spec. Hep. 6: 495. 1924. Schistochilaster s. (Steph.) H. Miller. Phỵtologia 20: 319. 1970.

G. tuloides (Hook. f. et Tayl.) Gott. et al., Synop. Hepat.: 620. 1846. Jungermannia t. Hook. f. et Tayl.. Londor Journ. Bot. 3: 558. 1844. Schistochila t. (Hook. f. et Tayl.) Steph.. Spec. Hep. 4: 89. 1909. Schistochilaster t. (Hook. f. et Tayl.) H. Miller. Phytologia 20: 320. 1970. Paraschistochila t. (Hook. f. et Tayl.) Schust.. Bull. Nat. Sci. Mus. Tokyo 14: 647. 1971.

= Gottschea ramulosa Col.. Trans. Proc. New Zealand Inst. 18: 243. 1886. Schistochila r. (Col.) Steph.. Spec. Hep. 4: 92. 1909. (Hodgson. 1941. p. 193).

-Gottschea.homophylla Col.. Trans. Proc. New Zealand Inst. 20: 250. 1888. (Hodgson. 1941. p. 194: "seems to be S[chistochila] tuloides").

G. viridis (Steph.) comb. nov.

Schistochila viridis Steph., Spec. Hep. 6: 496. 1924. Schistochilaster v. (Steph.) H. Miller. Phytologia 20: 320. 1970.

\section{Excludenda}

Included are the binomials of Gottschea and Schistochilaster not listed above. in alphabetical order of the epithets.

G. alata (Lehm.) Nees in Gott. et al.. Synop. Hepat.: 16. 1844 (Jungermannia a. Lehm. 1829). - Schistochila a. (Lehm.) Schiffn. 1893.

G. albistipula Col.. Trans. Proc. New Zealand Inst. 18: 242. 1886. "seems to be S[chistochila] repleta" (Hook. f. et Tayl. 1844) Steph. 1909. (Hodgson. 1941. pp. 184. 194).

G. appendiculata (Hook.) Nees in Gott. et al.. Synop. Hepat.: 14. 1844 (Jungermannia a. Hook. 1818). - Schistochila a. (Hook.) Trev. 1877.

G. balfouriana (Hook. f. et Tayl.) Gott. et al.. Synop. Hepat.: 622.1846 (Jungermannia b. Hook. f. et Tayl. 1844). = Schistochila b. (Hook. f. et Tayl.) Steph. 1909.

G. beccariana De Not. Memorie Real. Accad. Sci. Torino ser. 2. 28: 9. 1874. = Schistochila b. (De Not.) Trev. 1877.

G. berteroana (Hook.) Nees in Gott. et al.. Synop. Hepat.: 14. 1844 (Jungermannia b. Hook. 1829). - Schistochila b. (Hook.) Steph. 1909.

G. bicolor Col.. Trans. Proc. New Zealand Inst. 18: 240. 1886. "seems to be STchistochilal repleta" (Hook. f. et Tayl. 1844) Steph. 1909. (Hodgson. 1941. p. 194).

G. blumii (Nees) Nees in Gott. et al.. Synop. Hepat.: 19. 1844 (Jungermannia b. Nees 1823). = Schistochila b. (Nees) Trev. 1877.

G. carnosa Mitt.. Journ. Linn. Soc. Bot. (London) 15: 72. 1876. = Pachyschistochila c. (Mitt.) Schust. et Engel 1982.

G. chlorophylla Col.. Trans. Proc. New Zealand Inst. 18: 240. 1886. = Schistochila ciliata (Mitt. I854)

Steph. 1909. (Stephani. 1892. p. 274: Hodgson. 1941. p. 188: Hamlin. 1972. pp. 263. 333).

G. ciliata Mitt. in J. D. Hooker. F. Nov. Zel. 2: 151. 1854. = Schistochila c. (Mitt.) Steph. 1909. 
G. ciliistipula Col., Trans. Proc. New Zealand Inst. 19: 284. 1887. =Schistochila balfouriana (Hook. f. et Tayl. 1844) Steph. $1909(=$ S. marginata (Col. 1886) Steph. 1909). (Stephani, 1892, p. 274; Hodgson, 1941, p. 185).

G. clandestina Col., Trans. Proc. New Zealand Inst. 22: 454. 1890. =Schistochila repleta (Hook. f. et Tayl. 1844) Steph. 1909 (=S. heterodonta (Col. 1889) Steph. 1909. (Stephani, 1892, p. 274).

Schistochilaster colensoanus (Steph.) H. Miller, Phytologia 20: 318. 1970 ("colensoanum") (Schistochila c. Steph. 1909). = Pachyschistochila $c$. (Steph.) Schust. et Engel 1982.

G. compacta Coi., Trans. Proc. New Zealand Inst. 16: 349. 1884. "seems to be Stchistochila] colensoana Steph." 1909 (Hodgson, 1941, p. 194; Hamlin, 1972, p. 264).

G. compacta Col. (non Col. 1884, nec Col. 1887, p. 285), Trans. Proc. New Zealand Inst. 19: 284. 1887. $\approx$ G. laciniosa Col., Trans. Proc. New Zealand Inst. $21: 54$. 1889. $=$ Schistochila repleta (Hook. f. et Tayl. 1844) Steph. $1909(=S$. heterodonta (Col. 1889) Steph. 1909). (Stephani, 1892, p. 274; Hodgson, 1941, p. 184; Hamlin, 1972, pp. 264, 332).

G. compacta Col. (non Col. 1884, nec Col. 1887, p. 284), Trans. Proc. New Zealand Inst. 19: 285. 1887. $\equiv$ G. gregaria Col., Trans. Proc. New Zealand Inst. 21: 54. 1889. = Schistochila repleta (Hook. f. et Tayl. 1844) Steph. 1909 ( $=$ S. heterodonta (Col. 1889) Steph. 1909). (Stephani, 1892, p. 274; Hodgson, 1941, p. 184).

= Schistochila balfouriana (Hook. f. et Tayl. 1844) Steph. 1909. (Hamlin, 1972, pp. 264, 332).

G. dichotoma Col., Trans. Proc. New Zealand Inst. 18: 284. 1886. =Schistochila nobilis (Hook. 1818) Trev. 1877. (Stephani, 1909, p. 89; Hamlin, 1972, pp. 264, 333).

G. diplophylla (Hook. f. et Tayl. 1844) Nees in Gott. et al., Synop. Hepat.: 624. 1846. = Balantiopsis d. (Hook. f. et Tayl.) Mitt. 1884.

G. doriae De Not., Memorie Real. Accad. Sci. Torino ser. 2, 28: 10. 1874. =Schistochila d. (De Not.) Trev. 1877.

G. epiphyta Col., Trans. Proc. New Zealand Inst. 21: 57. 1889. =Schistochila balfouriana (Hook. f. et Tayl. 1844) Steph. 1909 (=S. marginata (Col. 1886) Steph. 1909). (Stephani. 1892, p. 274; Hodgson, 1941, p. 185).

G. erinacea (Hook. f. et Tayl. 1844) Nees in Gott. et al., Synop. Hepat.: 624. 1846. = Balantiopsis e. (Hook. f. et Tayl.) Mitt. 1867.

G. flavovirens Col., Trans. Proc. New Zealand Inst. 20: 248.1888 ("flavo-virens"). =Schistochila repleta (Hook. f. et Tayl. 1844) Steph. 1909 (=S. heterodonta (Col. 1889) Steph. 1909). (Stephani, 1892, p. 274; Hodgson, 1941, p. 184).

G. gayana Gott., Ann. Sci. Nat., Bot. ser. 4, 8: 320. 1857. = Schistochila g. (Gott.) Evans 1898.

G. glaucescens (Hook.) Nees in Gott. et al., Synop. Hepat.: 20. 1844 (Jungermannia g. Hook. 1818). ISchistochila g. (Hook.) Evans 1905.

G. gregaria Col., Trans. Proc. New Zealand Inst. 21: 54. 1889. =Schistochila repleta (Hook. f. et Tayl. 1844) Steph. 1909 (=S. heterodonta (Col. 1889) Steph. 1909). (Stephani, 1892, p. 274; Hodgson, 1941, p. 184).

=Schistochila balfouriana (Hook. f. et Tayl. 1844) Steph. 1909. (Hamlin, 1972, pp. 264, 332).

G. guttata Col., Trans. Proc. New Zealand Inst. 21: 53. 1889. = Schistochila repleta (Hook. f. et Tayl. 1844) Steph. 1909 (Hodgson, 1941, pp. 184, 193).

G. heterocolpos Col.. Trans. Proc. New Zealand Inst. 18: 239. 1886. =Schistochila repleta (Hook. f. et Tayl. 1844) Steph. 1909 (=S. heterodonta (Col. 1889) Steph. 1909). (Stephani, 1892, p. 274: Hodgson, 1941, p. 184).

G. heterodonta Col., Trans. Ptoc. New Zealand Inst. 21: 55. 1889. =Schistochila repleta (Hook. f. et Tayl. 1844) Steph. 1909. (Hodgson, 1941, pp. 184, 193).

G. hombroniana Mont., Ann. Sci. Nat., Bot. ser. 2, 19: 243. 1843. = Schistochila lehmanniana (Lindenb. 1832) Carring. et Pears. 1888. (Gottsche et al., 1844, p. 20).

G. jamesii Aust., Bull. Torrey Bot. Club 6: 302. 1879. = Schistochila laminigera (Hook. f. et Tayl. 1844) Evans 1892. (Hässel de Menendez, 1975, p. 7).

G. javanica Nees in Gott. et al., Synop. Hepat.: 19. 1844. = Schistochila sciurea (Nees 1830) Schiffn. 1893. (Buch, 1939, p. 16).

G. laciniosa Col., Trans. Proc. New Zealand Inst. 21: 54. 1889. = Schistochila repleta (Hook. f. et Tayl. 1844) Steph. 1909 (=S. heterodonta (Col. 1889) Steph. 1909). (Stephani, 1892, p. 274; Hodgson, 1941, p. 184).

G. laetevirens Col., Trans. Proc. New Zealand Inst. 18: 238. 1886 ("laete-virens"). -Schistochila unguicularis (Hook. f. et Tayl. 1844) Steph. 1909. (Hodgson, 1941, pp. 185, 193): 
-Schistochila balfouriana (Hook. f. et Tayl. 1844) Steph. 1909 (=S. marginata (Col. 1886) Steph. 1909). (Stephani. 1892, p. 274).

G. lamellara (Hook.) Nees in Gott. et al.. Synop. Hepat.: 20. 1844 (Jungermannia l. Hook. 1818) = Schistochila l. (Hook.) Evans 1892.

G. laminigera (Hook. f. et Tayl.) Gott. et al.. Synop. Hepat.: 623. 1846 (Jungermannia l. Hook. f. et Tayl. 1844). - Schistochila l. (Hook. f. et Tayl.) Evans 1892.

G. lehmanniana (Lindenb.) Nees in Gott. et al.. Synop. Hepat.: 20. 1844 (Jungermannia l. Lindenb. 1832). = Schistochila l. (Lindenb.) Carring. et Pears. 1888.

G. leucophylla Lehm. ex Gott. et al., Synop. Hepat.: 17. 1844. = Pachyschistochila l. (Lehm. ex Gott. et al.) Schust. et Engel 1982.

G. longiciliata Col., Trans. Proc. New Zealand Inst. 21: 54. 1889. =Schistochila balfouriana (Hook. f. et Tayl. 1844) Steph. 1909 (=S. marginata (Col. 1886) Steph. 1909). (Stephani. 1892. p. 274: Hodgson. 1941, p. 185).

G. longiseta Col.. Trans. Proc. New Zealand Inst. 21: 54. 1889. = Schistochila repleta (Hook. f. et Tayl. 1844) Steph. 1909 (=S. heterodonta (Col. 1889) Steph. 1909). (Stephani. 1892. p. 274: Hodgson. 1941, p. 184).

G.. macroamphigastra Col.. Trans. Proc. New Zealand Inst. 18: 238.1886 (in Bonner. 1966 wrongly as "macroamphigastriata"). $=$ Schistochila repleta (Hook. f. et Tayl. 1844) Steph. 1909 (=S. heterodonta (Col. 1889) Steph. 1909). (Stephani. 1892. p. 274: Hodgson. 1941. p. 184).

G. marginala Col.. Trans. Proc. New Zealand Inst. 18: $241.1886 .=$ Schistochila balfouriana (Hook. f. et Tayl. 1844) Steph. 1909. (Hodgson. 1941. pp. 185. 193).

G. mitteniana Col., Trans. Proc. New Zealand Inst. 21: 56. 1889. = Schistochila balfouriana (Hook. f. et Tayl. 1844) Steph. 1909. (Hodgson. 1941. pp. 185. 194).

G. moniliformis Col.. Trans. Proc. New Zealand Inst. 21: 56. 1889. = Schistochila appendiculata (Hook. 1818) Trev. 1877. (Stephani. 1892. p. 274: Hodgson. 1941. p. 183).

G. nitida Col.. Trans. Proc. New Zealand Inst. 18: 238. 1886. =Schistochila balfouriana (Hook. f. et Tayl. 1844) Steph. 1909 (=S. marginata (Col. 1886) Steph. 1909). (Stephani. 1892. p. 274: Hodgson. 1941, p. 185).

G. nobilis (Hook.) Nees in Gott. et al.. Synop. Hepat.: 21. 1844 (Jungermannia n. Hook. 1818). - Schistochila n. (Hook.) Trev. 1877.

G. pachyla (Hook. f. et Tayl.) Gott. et al.. Synop. Hepat.: 621.1846 (Jungermannia p. Hook. f. et Tayl. 1844). = Schistochila alata (Lehm. 1829) Schiffn. 1893. (Hässel de Menendez. 1975. p. 22: Schuster and Engel. 1977. p. 405).

G. pachyphylla (Lehm.) Nees in Gott. et al.. Synop. Hepat.: 19. 1844 (Jungermannia p. Lehm. 1834). - Pachvschistochila p. (Lehm.) Schust. et Engel 1982.

G. pallescens Col.. Trans. Proc. New Zealand Inst. 18: 241 . 1886. = Schistochila repleta (Hook. f. et Tayl. 1844) Steph. 1909 (=S. heterodonta (Col. 1889) Steph. 1909). (Stephani. 1892. p. 274: Hodgson. 1941. p. 184).

G. parvula Ángstr.. Ofvers. Kongl. Vet.-Akad. Förhandl. 29(4): 9. 1872. =Schistochila reflexa (Mont. 1845) Steph. 1909. (Schuster and Engel. 1975. p. 250. 1977. p. 357).

Schistochilaster piliger (Steph.) H. Miller. Phytologia 20:319.1970 ("piligerum.") = Schistochila p. Steph. 1890. According to Schuster and Engel. 1977, p. 405 this species belongs to Schistochila subg. Austroschistochila. not to Gottschea ( $=$ Schistochilaster).

G. pusilla Schiffn.. Forschungsreise S.M.S. "Gazelle." 4(Bot.): 3. 1890. =Schistochila alata (Lehm. 1829) Schiffn. 1893. (Hässel de Menendez. 1975. p. 22).

G. reflexa Mont.. Ann. Sci. Nat.. Bot. ser. 3. 4: 347. 1845. = Sihistochila r. (Mont.) Steph. 1909.

G. reinwardtii (Nees) Nees in Gott. et al.. Synop. Hepat.: 15. 1844 (Jungermannia $r$. Nees 1830). - Schistochila r. (Nees) Schiffn. 1898.

G. repleta (Hook. f. et Tayl.) Gott. et al.. Synop. Hepat.: 622. 1846 (Jungermannia r. Hook. t. et Tayl. 1844). = Schistochila r. (Hook. f. et Tayl.) Steph. 1909.

G. sciurea (Nees) Sande Lac. Ann. Mus. Bot. Lugd. Bat. I: 298. 1864 (Jungermannia s. Nees 1830). - Schistochila s. (Nees) Schiffn. 1893.

G. simplex Col.. Trans. Proc. New Zealand Inst. 18: 242. 1886. =Schistochila haltiouriana (Hook. f. et Tayl. 1844) Steph. 1909 (=S. marginata (Col. 1886) Steph. 1909). (Steohani. 1892. D. 274: Hamlin. 1972. pp. 265, 332).

-Schistochila unguicularis (Hook. f. et Tayl. 1844) Steph. 1909. (Hodgson. 1941. pp. 185. 193).

G. splachnophylla (Hook. f. et Tayl.) Gott. et al.. Synop. Hepat.: 621. 1846.(Jungermannia s. Hook. f. et Tayl. 1844). = Pachy'schistuchila s. (Hook. f. et Taỵl.) Schust. et Engel 1982. 
G. squarrosa Col., Trans. Proc. New Zealand Inst. 20: 248. 1888. =Schistochila nobilis (Hook. 1818) Trev. 1877. (Stephani, 1909, p. 89; Hodgson, 1941, p. 193).

G. stenocarpa Col., Trans. Proc. New Zealand Inst. 21: 55. 1889 ("steno-carpa"). "seems to. be S[chistochila] repleta" (Hook. f. et Tayl. 1844) Steph. 1909. (Hodgson, 1941, p. 194).

G. stratosa Mont., Ann. Sci. Nat., Bot. ser. 3, 4: 346. 1845. = Schistochila s. (Mont.) Evans 1892.

G. tahitensis Nadeaud, Enumer. Pl. Tahit.: 7. 1873. =Treubia $t$. (Nadeaud) Bescherelle 1898.

G. thwaitesii Mitt., Journ. Proc. Linn. Soc. Bot. 5: 101. 1861. - Schistochila t. (Mitt.) Steph. 1909.

G. trichotoma Col., Trans. Proc. New Zealand Inst. 18: 240. 1886. =Schistochila balfouriana (Hook. f. et Tayl. 1844) Steph. 1909 (=S. marginata (Col. 1886) Steph. 1909). (Stephani, 1892, p. 274; Hodgson, 1941, p. 185).

Schistochilaster truncatilobus (Steph.) H. Miller, Phytologia 20: 320.1970 (“truncatilobum"), =Schistochila t. Steph. in Rechinger, Denkschr. Kais. Akad. Wiss. Math.-Nat. KI. (Wien) 88: 34. 1913 (isotype in W!); syn. nov. =Schistochila samoana Steph. 1909 (isotype in W!).

G. truncatula Col., Trans. Proc. New Zealand Inst. 20: 247. 1888. =Schistochila balfouriana (Hook. f. et Tayl. 1844) Steph. 1909 (=S. marginata (Col. 1886) Steph. 1909). (Stephani, 1892, p. 274; Hodgson, 1941, p. 185).

G. unguicularis (Hook. f. et Tayl.) Gott. et al., Synop. Hepat.: 622. 1846 (Jungermannia u. Hook. f. et Tayl. 1844). = Schistochila u. (Hook. f. et Tayl.) Steph. 1909:

G. wallisii (Jack et Gott.) Bonner, Index Hepat. 6: 503.1966 (Schistochila w. Jack et Gott. 1892). =Schistochila blumii (Nees 1823) Trev. 1877. (Stephani, 1909, p. 80).

G. winkelmannii Col., Trans. Proc. New Zealand Inst. 21: 57. 1889. $=$ Schistochila appendiculata (Hook. 1818) Trev. 1877. (Stephani, 1892, p. 274; Hodgson, 1941, p. 183).

We wish gratefully to acknowledge the checking of the Gottschea protologue by Dr. F. Stafleu (Utrecht). He kindly confirmed that a generic description of Gottschea is lacking in Montagne (1843); however, the indirect reference to "section Aligerae" validates Gottschea Nees ex Mont. 1843.

\section{References}

Bonner, C. E. B. 1966. Index hepaticarum. 6: 481-739, Lehre (F. R. Germany).

Buch, H. 1939. Die Schistochila-Arten der Inseln Sumatra, Java und Celebes. Ann. Bryol. 12: 120.

Gottsche, C. M., J. B. G. Lindenberg and C. G. Nees von Esenbeck. 1844-1847. Synopsis hepaticarum. 834 pp., Meissner, Hamburg.

Grolle, R. 1966. Lebermoose aus Neuguinea, 4. Schistochila. Journ. Hattori Bot. Lab. 29: 238-252.

Hamlin, B. G. 1972. Hepaticae of New Zealand, I and II. Records Dominion Mus. (Wellington) 7: 243-366.

Hässel de Menendez, G. G. 1975. Noticias Hepaticologicas Sudamericanas 1-16. Revista Museo Argent. Cienc. Nat. "Bernardino Rivadavia," Bot. S(1): 1-26.

Hodgson, E. A. 1941. Review of the New Zealand species of Schistochila. Trans. Roy. Soc. New Zealand 71: 181-194.

Jones, E. W. 1976. African hepatics XXVIII. Schistochila Dum. Journ. Bryol. 9: 33-41.

Kitagawa, N. 1973. Miscellaneous notes on little-known species of Hepaticae, 26-50. Journ. Hattori Bot. Lab. 37: 263-273.

Miller, H. A. 1970. Some circum-Pacific Schistochilaceae. Phytologia 20: 315-323.

Montagne, C. 1843. Quatrième centurie ..., Déc. I-VI. Ann. Sci. Nat., Bot. ser. 2, 19: 238-266.

Reinwardt, K. G. K., K. L. Blume and C. G. Nees von Esenbeck. 1824. Hepaticae javanicae. Nova Acta Acad. Caes. Leop. Carol. Abt. 1, 12: 181-238, 409-417.

Schuster, R. M. 1963. Studies on antipodal Hepaticae I. Journ. Hattori Bot. Lab. 26: 185-309.

- 1968. Studies on antipodal Hepaticae X. Bull. Nat. Sci. Mus. (Tokyo) 11: 13-31. 14: 609-660.

and J. J. Engel. 1975. Austral Hepaticae V. Phytologia 30: 241-250.

- and -1977. Austral Hepaticae V. Journ. Hattori Bot. Lab. 42: 273-423.

$\longrightarrow$ and $\longrightarrow$ 1982. Austral Hepaticae XIII. Phytologia 50: 177-180.

Stephani, F. 1892. A revision of Colenso's Hepaticae. Journ. Linn. Soc., Bot. 29: 263-280.

1909-1910. Species hepaticarum 4: 65-100 [Schistochila], Genf. 